\title{
La marcha: diferencias según el sexo
}

\author{
S. NERÍN , A. VILLARROYA, E. CEPERO, \\ J. GÓMEZ, C. MARCO, M. MIGUELENA \\ Escuela Universitaria de Ciencias de la Salud \\ Universidad de Zaragoza
}

\begin{abstract}
Resumen
Objetivo: La finalidad de este trabajo ha sido valorar la movilidad de las articulaciones de la extremidad inferior y la actividad electromiográfica de los principales músculos de la misma, en un grupo de hombres y de mujeres, con la finalidad de establecer las diferencias entre ambos, al caminar por terreno llano y al ascender y descender escaleras.

Material y método: En el estudio han participado 22 hombres y 18 mujeres sanos a los que se ha determinado la movilidad articular con un sistema de análisis tridimensional denominado Orthobío y la actividad muscular mediante telemetría con un aparato MT8-3 Biological de MIE Medical Research Ltd y con con electrodos de superficie.

Resultado y discusión: Los resultados obtenidos muestran respecto a la movilidad articular, que para la marcha en terreno llano no hay diferencias significativas entre ambos grupos mientras que en el ascenso y descenso de escaleras se realizan algunas adaptaciones. Sin embargo respecto a la actividad muscular aparece una clara tendencia a una mayor actuación de los músculos en el grupo femenino, tanto al caminar por terreno llano como subiendo y bajando escaleras, sobre todo en los de marcada acción estabilizadora de la extremidad inferior. Este hecho puede deberse a las diferencias en la composición corporal, ya que las mujeres presentan un mayor porcentaje de masa grasa, por lo que, proporcionalmente, con su masa muscular tienen que movilizar un peso mayor.
\end{abstract}

Palabras clave: Electromiografía. Cinemática. Marcha en escaleras.

\section{Introducción}

La marcha es quizá uno de los movimientos que ha despertado más interés y sobre el que se han centrado numerosos trabajos a lo largo del tiempo, pero el avance constante de las técnicas de estudio y la incorporación de la informática en el registro y tratamiento de los datos, hace que el interés por la investigación de este tema no haya desaparecido con el fin de conocer con mayor precisión todos sus mecanismos y así, puede decirse, que la tecnología para el estudio de la marcha está continuamente cambiando. Pero, debe tenerse en cuenta que la marcha no se desarrolla siempre en terreno llano sino que debe adaptarse a todas las irregularidades del mismo y a las características de nuestro medio, en el que son sumamente frecuentes las escaleras, por lo que en la vida diaria es habitual la subida y bajada de las mismas.

Son abundantes los estudios existentes sobre la marcha normal en terreno llano, y mucho menos lo publicado acerca de la subida y bajada de escalera, pero dentro de todos ellos, y a pesar de que la mayoría de las veces se utilizan como muestra grupos de ambos sexos, no suelen darse datos de cada uno de dichos grupos de forma independiente. Únicamente, Kadaba y cols. ${ }^{1}$ compararan la marcha en llano entre hombres y mujeres, pero solamente en lo que se refiere a las amplitudes articulares a lo largo de ésta. Por ello, iniciamos el estudio de la marcha, valorando la movilidad de las articulaciones de la extremidad inferior y la 
actividad de los principales músculos de la misma, tanto caminando en llano como en ascenso y descenso de escaleras, en dos grupos, uno de hombres y otro de mujeres, con la finalidad de establecer las posibles diferencias entre ambos.

\section{Material y método}

En el estudio participaron 40 sujetos, con edades comprendidas entre los 18 y los 30 años (edad media 21,5 años), de los que 22 eran hombres (media de edad 21,8, altura $176 \mathrm{~cm}$ ) y 18 mujeres (media de edad 21,1, altura 162,3 cm), a los que únicamente se exigía no padecer alteración orgánica alguna que interfiriera con el normal desarrollo de la prueba.

A todos ellos se les realizó un análisis que consistía en la filmación de la marcha tanto en terreno llano como subiendo y bajando escaleras, para conocer las variaciones angulares a lo largo del movimiento, a la vez que se registraba la actividad electromiográfica de los principales músculos de la extremidad inferior.

Para el estudio de la marcha en llano se utilizó un tapiz rodante tipo EnTred, ya que la marcha en laboratorio, cuando se va a filmar con dos cámaras de vídeo fijas, reduce notablemente el espacio en el que se puede realizar el movimiento. Existen trabajos que ponen de manifiesto que las diferencias entre la marcha en suelo y la marcha en tapiz rodante son muy pequeñas 2,3,4,5 y consisten fundamentalmente en una mayor cadencia al realizar pasos más cortos, con periodos de oscilación algo más breves y periodos de apoyo más largos. Estas diferencias tienden a desaparecer tras un proceso de habituación al tapiz que varía de 7 minutos a 15 minutos $2,5,6$; en nuestro caso el tiempo de habituación fue de 10 minutos. La velocidad impuesta al tapiz fue de $3,5 \mathrm{Km} / \mathrm{h}$., ligeramente inferior a la considerada habitual para la marcha en suelo, pero debe tenerse en cuenta, como describieron Murray y col. ${ }^{3}$, que en la marcha en un terreno en movimiento disminuye la velocidad cómoda respecto a la realizada sobre suelo. La escalera en la que se realizó la prueba estaba formada por peldaños de $20 \mathrm{~cm}$ de altura y $25 \mathrm{~cm}$ de profundidad, y el ascenso y descenso se realizaba a velocidad libre.

El estudio de las imágenes de la marcha se realizó mediante un sistema de análisis tridimensional, denominado Orthobío 7 , formado por dos cámaras de vídeo, con las que se realizaba la filmación de las pruebas, un calibrador o sistema de referencia, que sirve para relacionar las coordenadas reales del espacio respecto a él mismo, marcadores, que son unas semiesferas sólidas de $1,5-2 \mathrm{~cm}$ de diámetro, recubiertas de material reflectante con el fin de que destaquen en la filmación y que se pueda realizar la digitalización automática, y un soporte informático que analiza estas imágenes.

Los marcadores se colocaron en: espina ilíaca ánterosuperior, trocánter mayor, cóndilo externo femoral, maléolo externo y base de quinto metatarsiano. La unión de estos marcadores delimitaban segmentos entre los cuales se valoraban los ángulos de cadera, rodilla y tobillo.

Tras la filmación, se analizó un ciclo central de la marcha en cada una de las pruebas realizadas. Por visualización directa de lo filmado en el ordenador, se escogían unos puntos concretos del ciclo de marcha que servían para dividirlo en fases. Estos puntos correspondían a la primera imagen de los siguientes momentos (Figura 1):

(CT) - Contacto de talón derecho.

(PP) - Pie plano derecho

(AU) - Despegue de pie izquierdo

(DT) - Despegue de talón derecho

(AB) - Contacto de talón izquierdo

(DA) - Despegue de antepié derecho

(AV) - Avance del miembro inferior derecho al oscilar. En este caso la imagen tomada no era la primera sino que correspondía al momento en que el pie derecho cruzaba por delante del miembro inferior izquierdo en apoyo.

(CT) - Nuevo contacto de talón derecho.

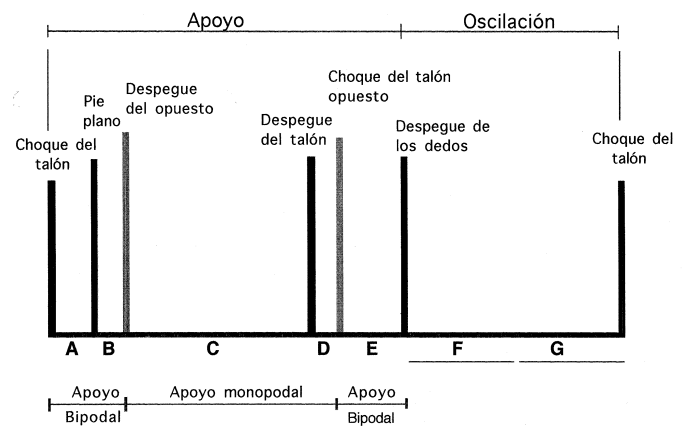

Figura 1. Fases en las que se ha dividido el ciclo de marcha

Entre estos momentos quedaron delimitadas una serie de fases que se nombraron alfabéticamente (Figura 1). Estas fases son:

\section{Durante el primer doble apoyo:}


Fase A - Desde que contacta el talón del pie derecho en el suelo hasta el apoyo completo de éste.

Fase B - Desde el apoyo completo de la planta del pie derecho hasta el despegue del pie izquierdo.

Durante el primer apoyo unilateral o periodo portante:

Fase C - Desde el despegue del pie izquierdo hasta el despegue de talón derecho.

Fase D - Desde el despegue de talón derecho hasta el contacto en el suelo del pie izquierdo.

\section{Durante el segundo doble apoyo:}

Fase E - Desde el contacto de talón izquierdo hasta el despegue del antepié derecho.

Durante el segundo apoyo unilateral o periodo oscilante:

Fase F - Desde el despegue de antepié derecho hasta que el pie cruza por delante del miembro inferior izquierdo en apoyo.

Fase G - Desde el cruce del pie derecho hasta el nuevo contacto del talón.

El ciclo de ascenso y descenso de escaleras quedó dividido de la misma forma, con la salvedad de que en el ascenso, como se accede al escalón superior generalmente con el pie plano o sólo con el antepié, no existe la fase previa de contacto de talón, mientras que en el descenso se invierte la primera fase respecto a la marcha en terreno llano ya que el contacto del pie con el escalón inferior sigue la secuencia antepié-talón.

Los músculos estudiados fueron: glúteo mayor, glúteo medio, recto anterior, vasto interno, bíceps femoral, gemelo externo y tibial anterior del miembro inferior derecho y el registro electromiográfico de su actividad se llevó a cabo mediante el sistema telemétrico MT8-3 Biological de MIE Medical Research Ltd., utilizando para la recogida electrodos de superficie, que se colocaron sobre la parte central del vientre muscular, como indica Clarys 8 , en línea con las fibras musculares y con una distancia interelectrodo de unos $2 \mathrm{~cm}$.

Para cuantificar las señales electromiográficas obtenidas se realizó una integración de las mismas, pasando por tres fases sucesivas:
- Rectificación de la señal convirtiendo los valores negativos en positivos, (onda por encima de cero en la gráfica).

- Filtrado de las frecuencias inferiores a $10 \mathrm{~Hz}$, como proponen Cipriani y cols., Koh y Grabiner y Tata y cols. 9,10,11, entre otros, ya que eliminan ruidos y no se pierden prácticamente potenciales de unidad motora 12.

- Integración de la señal cada 0,025 segundos.

Para poder trabajar con los datos obtenidos se necesita normalizarlos, es decir, expresarlos en porcentaje de un valor de referencia generado por los mismos electrodos. En este caso, se utilizó como valor normalizador el pico máximo de la actividad recogida durante una contracción isométrica máxima. Este valor se propone como normalizador en muchos estudios dinámicos, y entre ellos en los de la marcha 13,14,15,16,11,17.

El trazado electromiográfico de la marcha en llano y durante la subida y bajada de escalera se dividió en los segmentos correspondientes a las distintas fases del ciclo de la marcha, antes comentadas, y se calculó qué porcentaje suponían los valores obtenidos en cada una de ellas con respecto al valor normalizador.

El tratamiento de los datos recogidos se efectuó mediante el programa "Stat-View SE+Graphics", utilizando, para comparar la movilidad articular entre el grupo de hombres y el de mujeres, el T-test para series no apareadas, ya que la distribución de los datos era normal, y para la comparación de la actividad muscular entre ambos grupos, al no ser una distribución normal, el test de la "U" de MannWhitney que ordena las variables y les atribuye rangos. En todos los tests de comparación el valor del estadístico obtenido se asoció a una probabilidad, trabajando con un nivel de significación del 0,05 .

\section{Resultados y discusión}

\section{Datos cinemáticos}

En terreno llano, y de acuerdo con Kadaba y cols. $^{1}$, no hemos encontrado diferencias significativas entre los grupos de hombres y mujeres en la movilidad articular de las articulaciones de la 

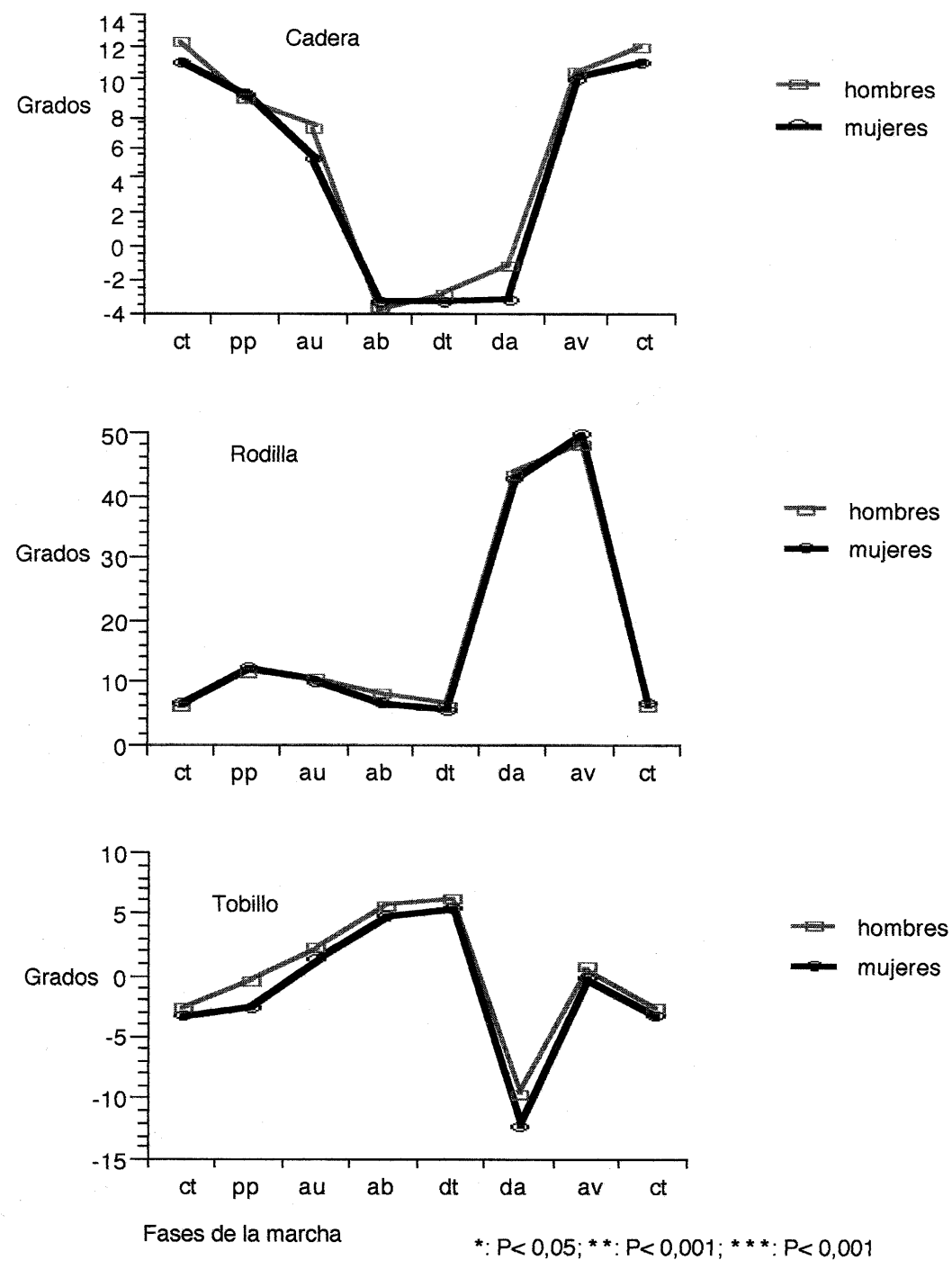

Figura 2. Movilidad de las articulaciones de cadera, rodilla y tobillo a lo largo del ciclo de marcha en terreno llano, en hombres y mujeres.

extremidad inferior, como se puede apreciar en la Figura 2 .

Sin embargo, sí que las hay en el ascenso y descenso de escaleras, debidas probablemente a la diferencia de talla entre ambos grupos, motivo por el que se deben realizar adaptaciones articulares para compensar dicha diferencia, ya que la distancia a cubrir entre escaleras es igual para todos. Así, en el ascenso de escalera (Figura 3), se aprecia durante el despegue del pie del suelo, una mayor extensión de la cadera (p: 0,01$)$ y de la rodilla (p: 0,01) en las mujeres que en los hombres, así como una tendencia a mayor extensión también en el tobillo, para intentar alargar más la extremidad inferior y que el otro miembro acceda al peldaño superior. En el contacto del pie con dicho escalón, el tobillo tiende a estar algo más extendido (p: 0,03) en las mujeres, probablemente porque, a pesar de las compensaciones del otro miembro intentando alargarlo, el cuerpo no va lo suficiente hacia delante quedando la tibia algo más hacia atrás, con un ángulo mayor en el tobillo. En el descenso (Figura 4 ), se produce una mayor extensión de cadera (p: $0,05)$ y rodilla (p: 0,001$)$, cuando se contacta con el escalón inferior, también con la finalidad de alargar la extremidad y acceder al mismo. 

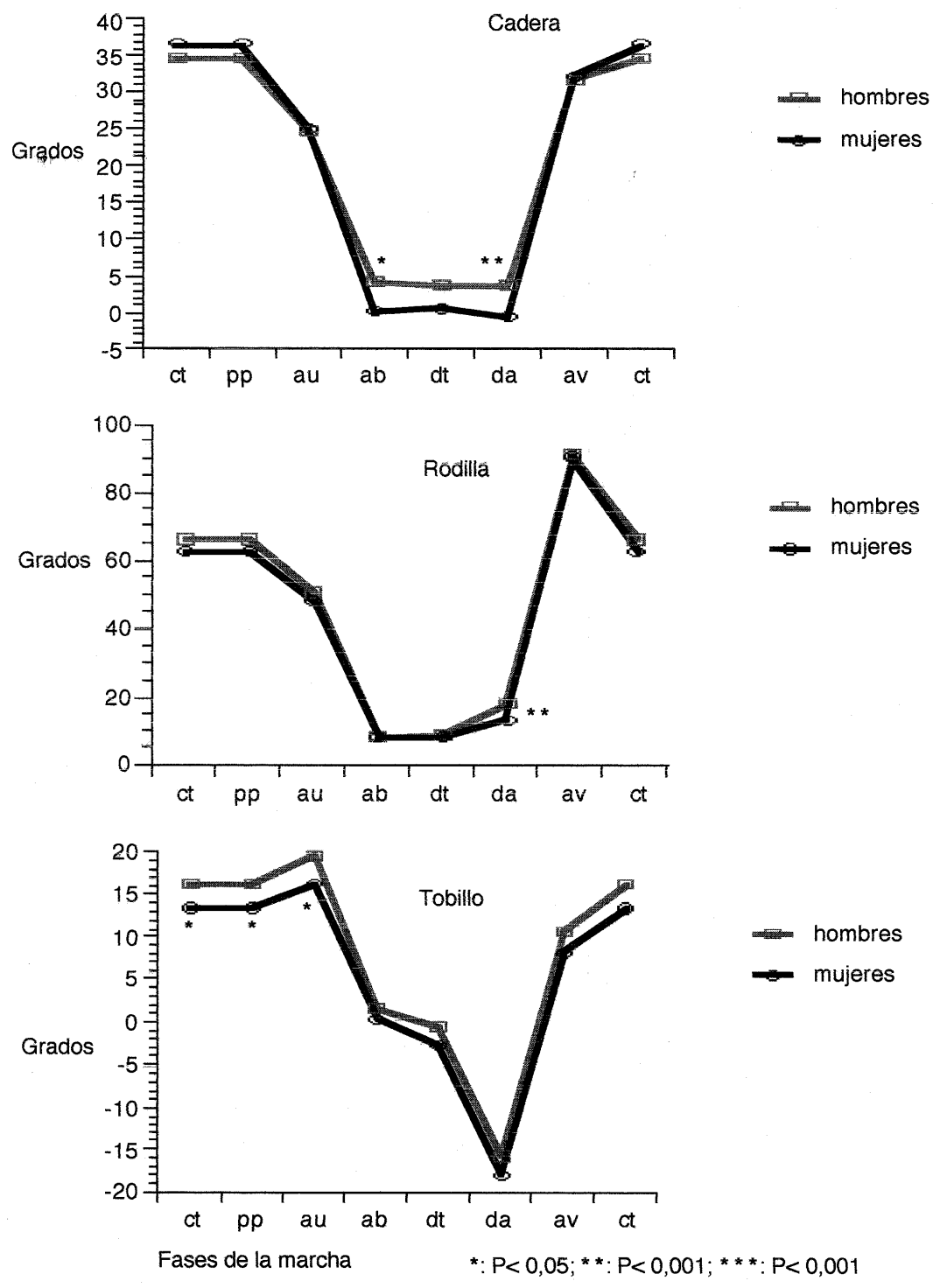

Figura 3. Movilidad de las articulaciones de cadera, rodilla y tobillo al ascender escaleras, en hombres y mujeres.

Así, como vemos, se tiende a compensar la menor altura, y por tanto menor longitud de las extremidades inferiores, del grupo de las mujeres intentando alargar, en el ascenso, la extremidad posterior de impulso, para permitir que la otra llegue al escalón superior, y en el descenso, la extremidad anterior que accede al nivel inferior.

\section{Actividad muscular}

Por lo que respecta al Glúteo mayor (Figura 5), al caminar por terreno llano, presenta su máxima acción en la fase inicial de apoyo para evitar la flexión de la cadera que tiende a producirse tras el choque de talón 12,18,19,20. Al ascender su actividad es significativamente mayor $21,22,15$, puesto que debe realizar una importante extensión de cadera que mantenga todo el cuerpo por encima del miembro inferior portante, siendo máxima al inicio del apoyo. En el descenso, la actividad del glúteo mayor es mucho menor que en el ascenso y similar a la encontrada en la marcha en llano, presentando su actuación máxima en la fase de apoyo monopodal 15,23 , controlando la flexión de cadera que ocurre cuando se está produciendo el descenso del otro miembro hacia un escalón inferior. Al comparar los valores medios de la actividad de este músculo por sexos, vemos que, en terreno llano, las mujeres presentan una tendencia a desarrollar una actividad mayor en las fases iniciales del apoyo que es cuando tiene su mayor actuación. 

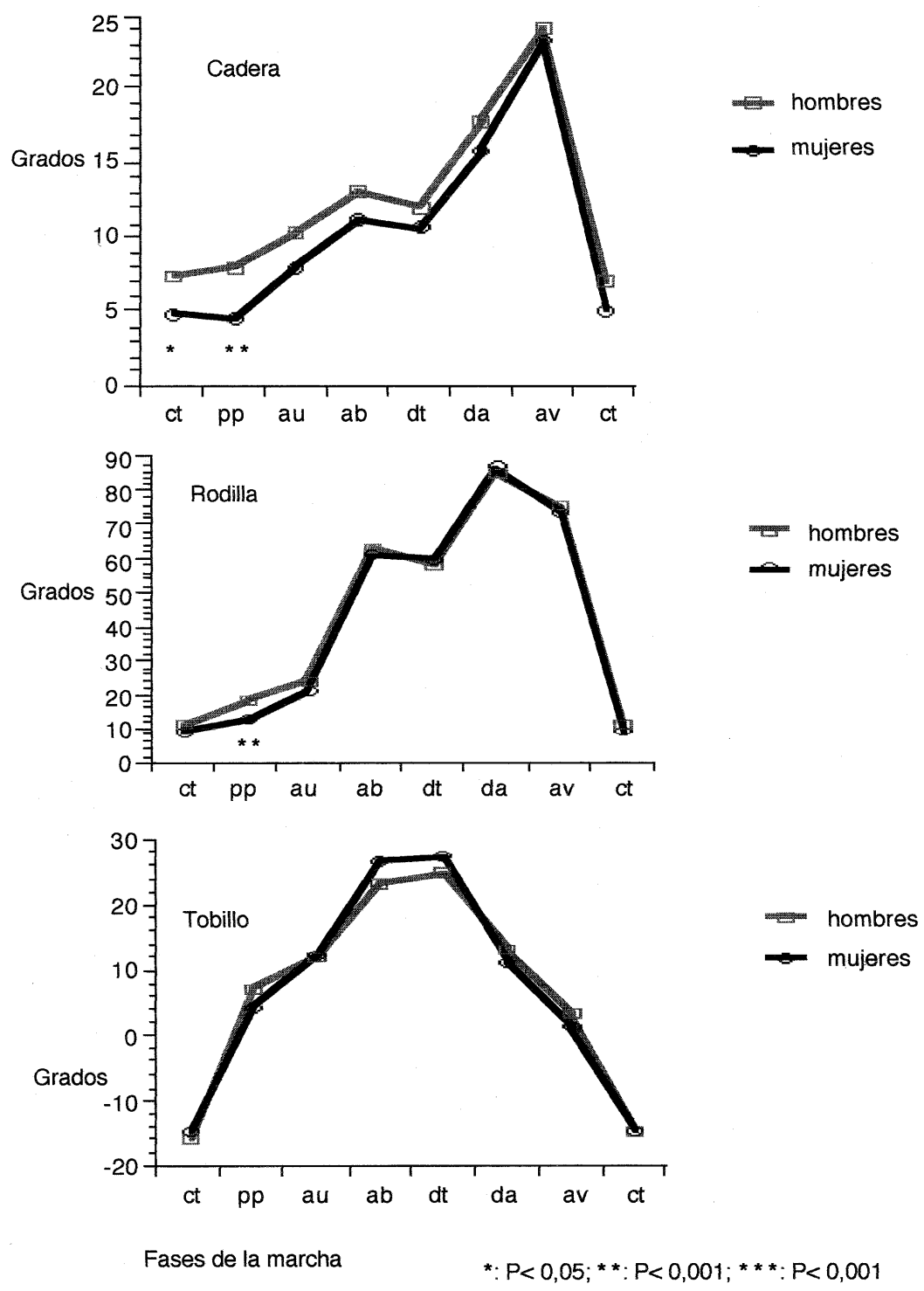

Figura 4. Movilidad de las articulaciones de cadera, rodilla y tobillo al descender escaleras, en hombres y mujeres.

En el ascenso de escaleras esta actividad es claramente superior a lo largo de todas las fases del ciclo, principalmente durante el apoyo que es cuando mayor es su participación, siendo significativa la diferencia en la mayoría de ellas. Al descender, también se aprecia una mayor actividad en el grupo de sexo femenino, pero solamente en el inicio del apoyo.

En su acción extensora, el glúteo mayor se ve ayudado por el bíceps femoral (Figura 6) que en terreno llano presenta actividad, como se describe habitualmente 24,25,13,14, en el momento del contacto de talón en el suelo para colaborar con el glúteo mayor en la extensión de cadera que se necesita en este momento y para controlar la acción del cuadriceps, que actúa para frenar la flexión que se tiende a producir por el peso del cuerpo en este apoyo. Presenta un segundo pico de actividad al final de la oscilación para controlar la flexión de cadera y frenar el lanzamiento inercial de la pierna, decelerando el talón antes del contacto con el suelo 26,27,28. En el ascenso, presenta también dos picos de actividad que se producen, el primero, igual que en llano, en el contacto del pie con el escalón, con la misma finalidad pero con una intensidad mucho mayor $15,13,23$, y el segundo 


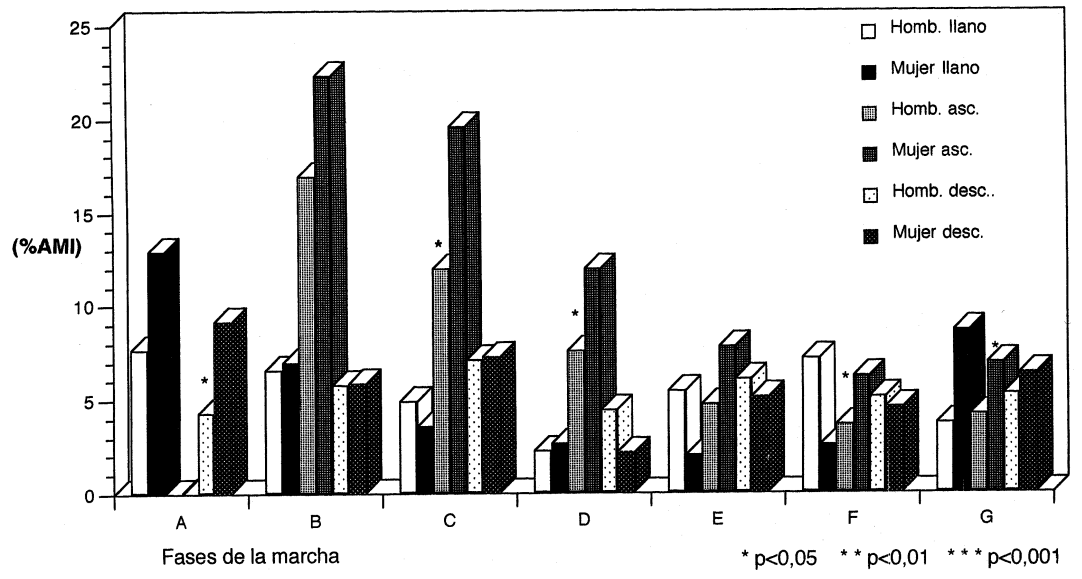

Figura 5. Actividad electromiográfica del glúteo mayor, al caminar por terreno llano, ascender y descender escaleras, en hombres y mujeres.

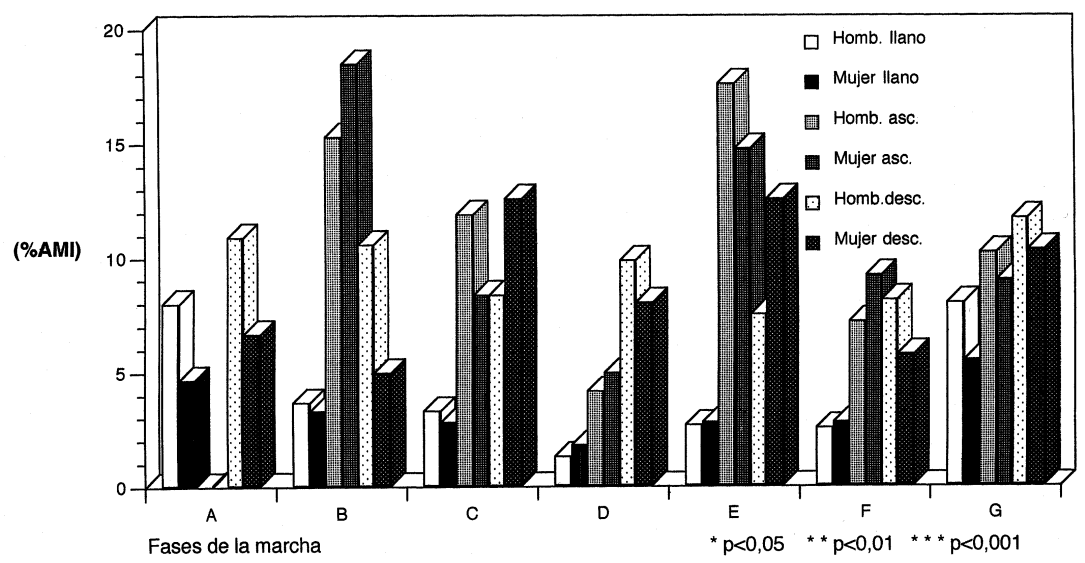

Figura 6. Actividad electromiográfica del bíceps femoral, al caminar por terreno llano, ascender y descender escaleras, en hombres y mujeres.

lo describen Cicotti y cols. ${ }^{13}$ y Joseph y Watson 23 durante la oscilación,pero nosotros también hemos encontrado actividad, al igual que Lyons y cols. 15 en el momento del despegue del pie para ayudar en el impulso de la extremidad inferior. Al descender, el bíceps femoral actúa de un modo muy continuado a lo largo de todo el ciclo, en primer lugar durante todo el apoyo 13,29 , controlando, por una parte, la flexión de cadera que ocurre cuando el miembro inferior opuesto va descendiendo, y por otra, junto con el cuadriceps, la posición de la rodilla, pero, además, tiene un papel esencial en la estabilización lateral y rotacional de esta articulación en un momento en que, por encontrarse flexionada, la actuación ligamentosa es mínima. Más tarde, continúa actuando durante la oscilación, controlando anteroposteriormente el miembro inferior. A diferencia de lo que ocurre en el glúteo mayor, en este músculo no se aprecian diferencias claras en su actividad entre los dos grupos analizados.

El Glúteo medio (Figura 7) actúa en los tres tipos de marcha estudiadas en la fase de apoyo y con la misma finalidad, la de evitar la caída de la pelvis hacia el lado opuesto y colaborar en el equilibrio transversal del miembro inferior 22,15,26,21,30. En el ascenso de escalera, presenta una actividad significativamente mayor que en terreno llano y que en el descenso ya que, además, debe colaborar en la potente extensión de cadera que se precisa para el ascenso. A diferencia de la marcha en llano y al igual que en el descenso, 
continúa con una actividad moderada durante la oscilación con la finalidad de controlar trasversalmente la extremidad inferior, junto con los músculos aductores, en su camino hacia el escalón superior o inferior. Como se aprecia en la Figura 7, tanto en llano como en escalera, prácticamente en todas las fases, los valores medios de actividad eléctrica son mayores en el grupo de mujeres que en

el de los hombres.

El cuadriceps (Figuras 8 y 9) actúa muy poco en la marcha en terreno llano, sobre todo el recto anterior, y presenta su pico de actuación en el momento del contacto de talón con el suelo para evitar la flexión pasiva de la rodilla que se produce al cargar el peso del cuerpo 13,14,31,32 .

Sin embargo, en el ascenso, este músculo tiene un papel esencial desde el contacto del pie en el escalón, para extender la rodilla y mantenerla así durante el apoyo monopodal 33,13,23,11, siendo también mayor la acción del vasto interno que la del recto anterior, ya que presenta una desventaja mecánica importante al encontrarse la cadera muy flexionada. Al igual que en llano, las dos porciones presentan una moderada actividad durante la oscilación, el recto anterior para colaborar en la flexión de cadera y las dos controlando, con los isquiotibiales, la flexión pasiva de rodilla que se produce en la oscilación, secundaria a la de la cadera, para acceder al escalón superior.

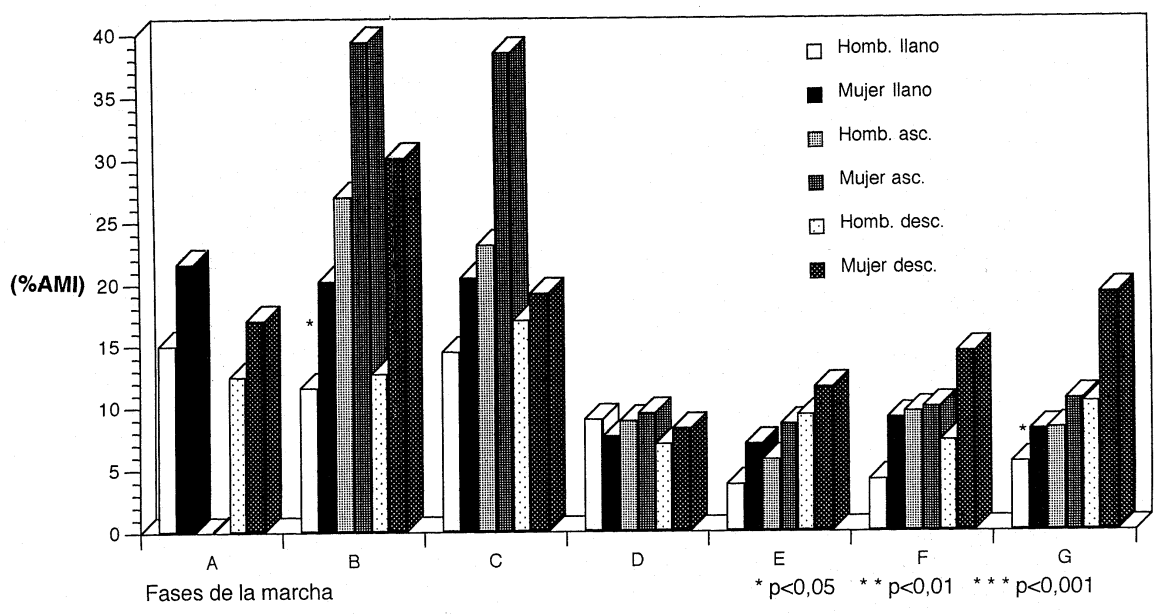

Figura 7. Actividad electromiográfica del glúteo medio, al caminar por terreno llano, ascender y descender escaleras, en hombres y mujeres.

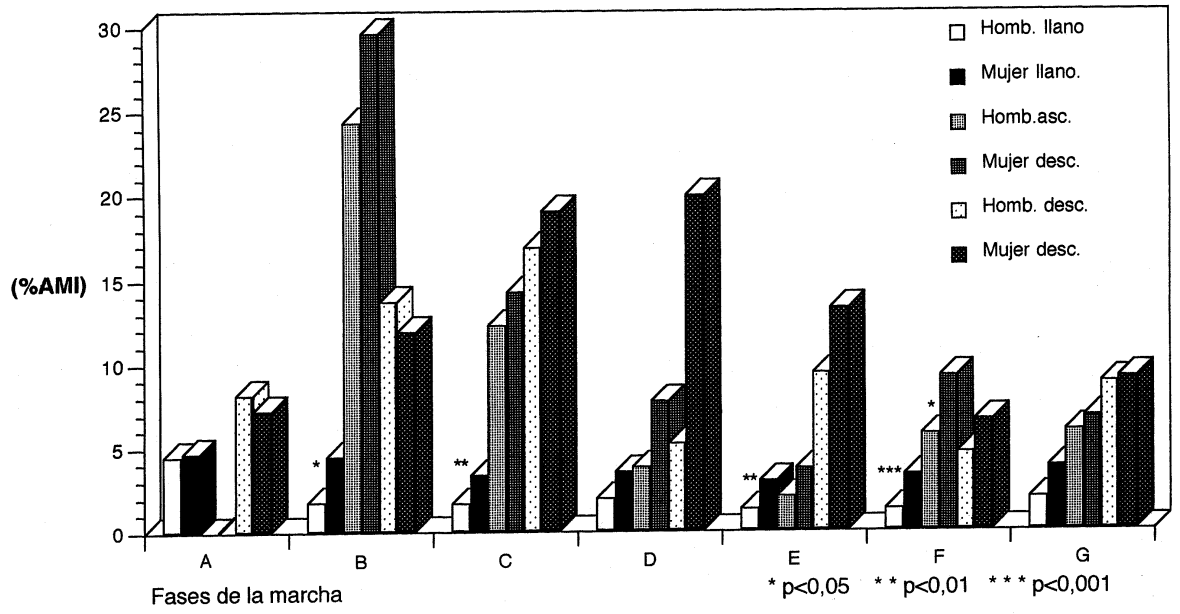

Figura 8. Actividad electromiográfica del recto anterior, al caminar por terreno llano, ascender y descender escaleras, en hombres y mujeres. 


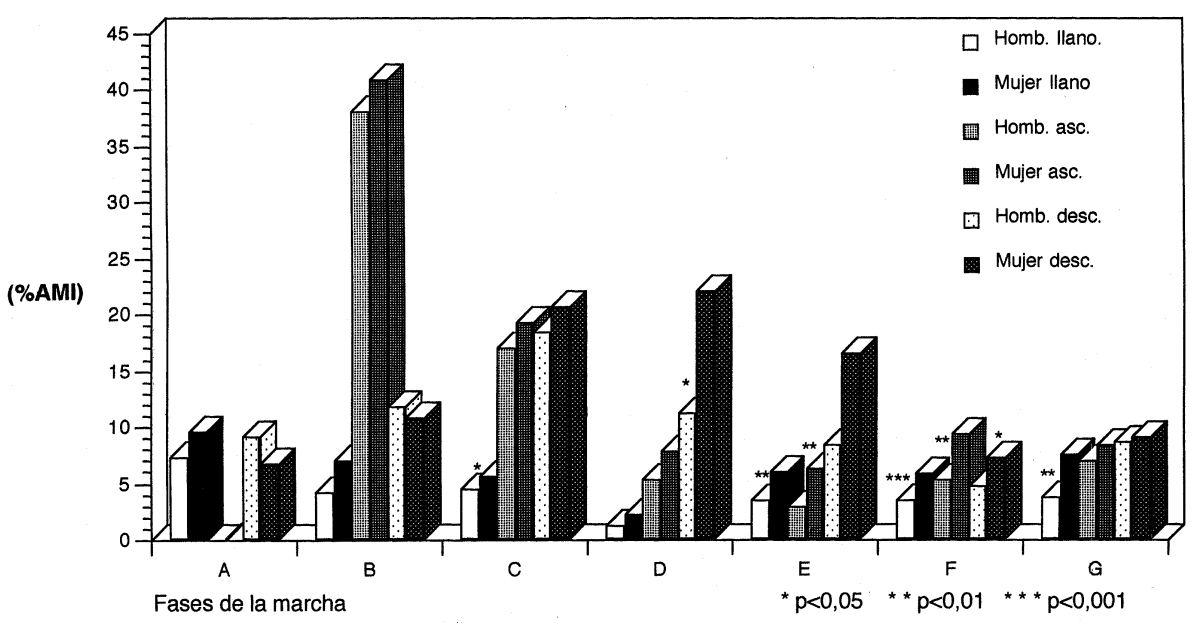

Figura 9. Actividad electromiográfica del vasto interno, al caminar por terreno llano, ascender y descender escaleras, en hombres y mujeres.

$\mathrm{Al}$ descender, estos músculos presentan su pico de acción en el apoyo monopodal 13,22,21, con una intensidad mayor que en llano, ya que se necesita una potente contracción excéntrica para regular la flexión de rodilla que ocurre mientras el miembro inferior opuesto desciende hacia el escalón inferior. Esta actividad persiste, aunque con menor intensidad, cuando se abandona el escalón ya que, tras la primera parte de la oscilación, se produce una extensión de rodilla para acceder al nuevo peldaño.

Se aprecia cómo también en estos músculos aparece, durante la marcha en terreno llano, mayor actividad en el grupo de las mujeres, a lo largo de todo el ciclo, con diferencias significativas respecto a los valores de los hombres en los momentos en que más actúan (fase de apoyo monopodal y despegue del pie e inicio de la oscilación). En el ascenso y descenso de escaleras persiste esta tendencia ya que en todas las fases hay una actividad muscular media mayor en el grupo femenino que en el masculino, siendo significativas las diferencias en algunas fases, como se aprecia en las Figuras 8 y 9 .

El gemelo externo (Figura 10) al caminar en terreno llano comienza a actuar, como se describe clásicamente, cuando se encuentra el pie plano en el suelo y se está progresando hacia delante, controlando, mediante una contracción de tipo excéntrico, el avance de la tibia; más tarde, su actividad aumenta, ya que realiza una contracción isométrica para solidarizar la tibia con el pie y conseguir el despegue de talón y, por último, mediante una contracción concéntrica, eleva el pie del suelo $30,34,31,32$.
Al ascender, muestra una actividad significativamente mayor que en llano durante todo el ciclo, colaborando, desde que contacta el pie en el escalón y se hace cargo de la progresión, en la extensión de todo el miembro inferior; su actividad desciende mucho en el momento en el que el otro miembro apoya en el escalón y es quien se hace cargo de la progresión. Al descender, los gemelos actúan ya desde el apoyo del pie en el escalón, pero sobre todo durante el apoyo unipodal, cuando se está produciendo la triple flexión del miembro inferior que apoya, controlando la inclinación anterior de la tibia mediante una contracción de tipo excéntrico, aunque su acción es significativamente menor que en llano y que en ascenso; posteriormente, aunque mucho menos, siguen actuando durante la oscilación, ya que el acceso al peldaño inferior se realiza con el pie en extensión $y$, a pesar de que se ve favorecida por la acción de la gravedad, no es una caída pendular sino muscularmente controlada junto con la acción del tibial anterior.

Los gemelos también siguen la tendencia general a presentar una mayor actuación en las mujeres que en los hombres en todas las fases de las tres situaciones estudiadas, siendo sus diferencias significativas en muchas de ellas, sobre todo en las que mayor acción presentan, apoyo monopodal, despegue del pie y oscilación, en llano $\mathrm{y}$ ascenso, y apoyo monopodal y despegue del pie, en el descenso.

Por último, el tibial anterior (Figura 11) presenta en la marcha en terreno llano dos picos de actividad clásicamente descritos $13,14,26,21$. 


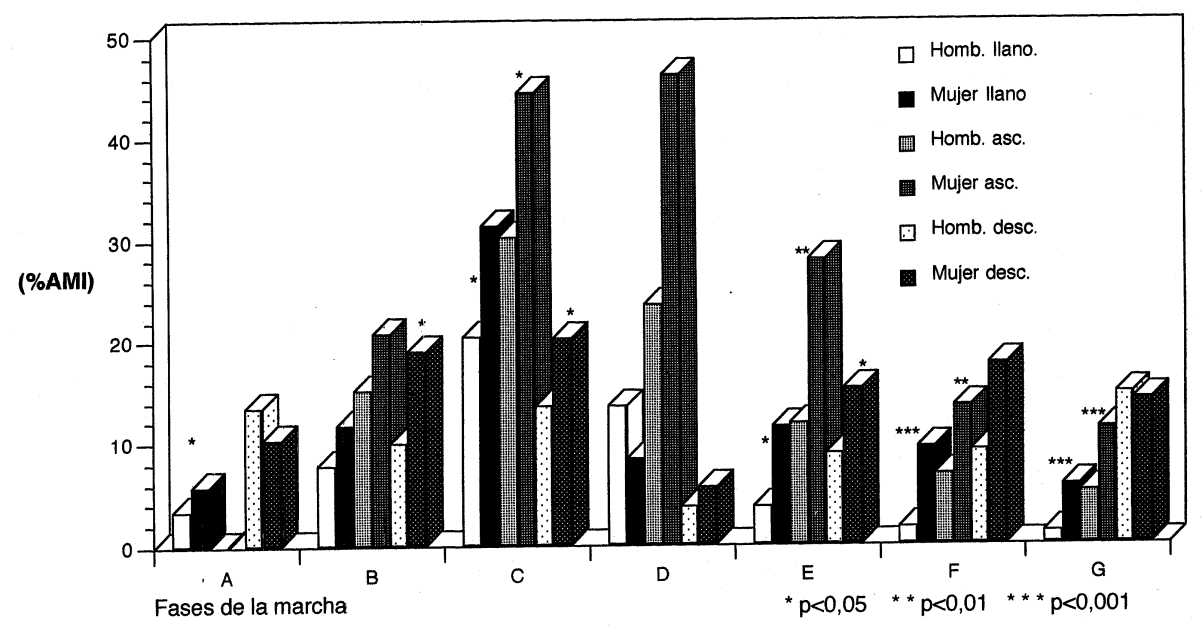

Figura 10. Actividad electromiográfica del gemelo externo, al caminar por terreno llano, ascender y descender escaleras, en hombres y mujeres.

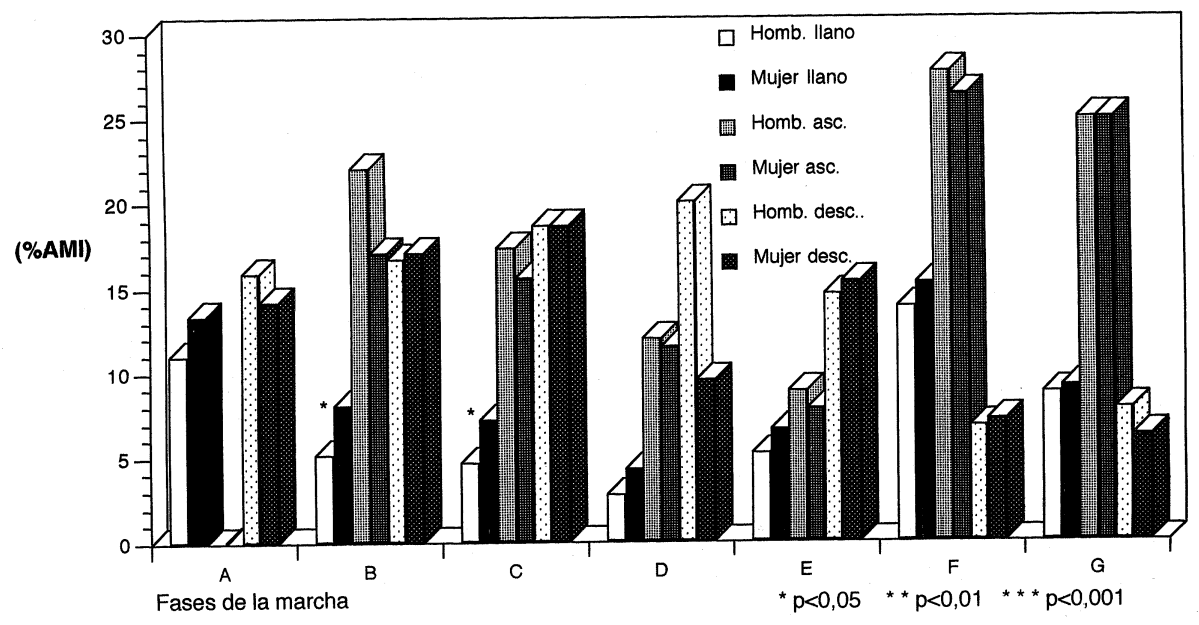

Figura 11. Actividad electromiográfica del tibial anterior, al caminar por terreno llano, ascender y descender escaleras, en hombres y mujeres.

El primero, en el momento del contacto de talón, para controlar, mediante una contracción de tipo excéntrico, el abatimiento del pie en el suelo, aunque su actividad, en nuestro trabajo, no es todo lo elevada que se esperaría, ya que la marcha en tapiz hace que al producirse el contacto de talón en el suelo se vea enseguida arrastrado hacia atrás y, por tanto, no precise de un control tan importante del tibial anterior.

El segundo pico se produce tras el despegue del antepié para elevar la punta del pie y evitar su choque con el suelo, colaborando en el acortamiento del miembro inferior. En el ascenso de escaleras, su acción es significativamente mayor que en terreno llano, siendo muy continua a lo largo del ciclo, de forma que trabaja durante todo el apoyo para mantener, junto con los gemelos, la estabilidad anteroposterior del pie, y presentando sus picos de actividad durante la oscilación $33,29,23$, para realizar la flexión que le permita acceder al escalón superior evitando el roce con el mismo. En el descenso, el tibial anterior también presenta una actividad muy mantenida a lo largo de todo el apoyo y hasta el despegue del pie, colaborando con los gemelos en la estabilización anteroposterior, como en el ascenso, mientras el miembro inferior se hace cargo del equilibrio y de la progresión al siguiente nivel, pero durante la oscilación, esta actividad ${ }^{13}$, es muy ligera, puesto que sólo controla la acción de los gemelos ya que el acceso al nuevo escalón se produce con el tobillo en extensión. En este músculo, como en el bíceps femoral, no se aprecia una tendencia en el sentido 
de que actúe más en un grupo que en otro en las diferentes fases del ciclo de la marcha, ni caminando en terreno llano, ni en escalera.

Por tanto, se aprecia cómo, en general, existe una clara tendencia a una mayor actividad muscular en el grupo femenino, estadísticamente significativa en muchas de las fases del ciclo de marcha, tanto caminando en terreno llano como subiendo o bajando escaleras, en los músculos con marcada acción estabilizadora del miembro inferior, glúteos mayor y medio, cuadriceps y gemelos. Sin embargo, es diferente la actuación de otros músculos como el bíceps femoral y el tibial anterior en los que los valores son irregulares y no existe una tendencia clara ni diferencias que sean significativas entre ambos grupos de estudio. No parece que estas variaciones en la actividad se deban a las diferencias en la altura, ya que, aunque en algunos casos, debido a esto, se necesita realizar movimientos articulares más amplios que podrían implicar una mayor actividad muscular para colaborar en ellos, esta mayor actividad se produce, en los músculos citados, al caminar en llano, donde no existen diferencias significativas en las amplitudes articulares entre hombres y mujeres, $\mathrm{y}$ en prácticamente todas las fases subiendo y bajando escaleras, y no sólo en las que la movilidad articular es diferente. El hecho de que las mujeres necesiten un mayor esfuerzo muscular puede deberse a las diferencias en la composición corporal, ya que, como es sabido 35,36,37 presentan un mayor porcentaje de masa grasa que los hombres, por lo que, proporcionalmente, con su masa muscular tienen que movilizar un peso mayor, es decir, la grasa corporal puede influenciar la carga que se tiene que soportar durante la marcha, ya que es un peso inerte que debe ser trasportado por el esfuerzo de los músculos 38 .

\section{Bibliografía}

1.- Kadaba MP, Ramakrishnan HK, Wootten ME. Measurement of lower extremity Kinematics during level walking. J Orthop Res 1990; 8: 383-392.

2.- LLana S, Brizuela G. Modificaciones en la cinética de la marcha humana inducidas por el treadmill. Selección 1996; 5: 166-170.

3.- Murray MP, Spurr GB, Sepic SB, Gardner GM, Mollinger LA. Treadmill vs floor walking: Kinematics, electromiogram and heart rate. J Appl Physiol 1985; 59: 87-97.

4.- Van Ingen Schenau GJ. Some fundamental aspects of biomechanics of overground versus treadmill locomotion. Medicine and Science in Sports and
Exercise, 1980; 12: 257-261.

5.- Wall JC, Charteris J. The process of habituation to treadmill walking at different velocities. Ergonomics 1980; 23: 425-435.

6.- Wall JC, Charteris J. A kinematic study of long-term habituation to treadmill walking. Ergonomics 1981; 24: 531-542.

7.- Villarroya A, Aguilar JJ, Torres F, Asirrón PJ. Orthobío: un nuevo sistema de análisis del movimiento en tres dimensiones. Rehabititación 1997; 31: 265-272.

8.- Clarys JP. A review of EMG in swimming, expanation of facts and/or feedback information. En: Hollander AP, Huying PA, De Groot G eds. Swimming Science IV. Champaing: Human Kinetics, 1983; 123-125.

9.- Cipriani DJ, Armstrong CW, Gaul S. Backward walking at three levels of treadmill inclination: an electromyographic and kinematic analysis. JOSPT 1995; 22: 95-102.

10.- Koh TJ, Grabiner MD. Cross talk in surface electromyograms of human hamstring muscles. J Ortop Res 1992; 10: 701-709.

11.- Tata JA, Peat M, Grahame RE, Quanbury AO. The normal peak of electromyographic activity of the quadriceps femoris muscle in the stair cycle. Anat Anz 1983; 153: 175-188.

12 .- Basmajian JV, De Luca CJ. Muscles alive. 5th ed. Baltimore: Williams \& Wilkins, 1985.

13.- Cicotti MG, Kerlan RK, Perry J. An electromyographic analysis of the knee during funcional activities. Am J Sports Med 1994; 22: 645-650.

14.- Dubo HI, Peat M, Winter DA, Quanbury AO, Hobson DA, Steinke T, Reimer G. Electromyographic temporal analysis of gait: Normal human locomotion. Arch Phys Med Rehab 1976; 57 : 415-418.

15.- Lyons K, Perry J, Gronley JK, Barnes L, Antonelli D. Timing and relative intensity of hip extensor and abductor muscle action during level and stair ambulation. An EMG study. Phys Ther 1983; 63: 1597-1605.

16.- Perry J. Schmidt C, Antonelli DJ. Surface versus intramuscular electrodes for electromyography of superficial and deep muscles. Phys Ther 1981; 61 (1): 7-15

17.- Winter DA, Yack HJ. EMG profiles during normal human walking: stride-to-stride and inter-subject variability. Electroencephalogr Clin Nuerophysiol 1987; 67: 402-411.

18.- Corcoran PJ, Peszczynsky M. La marcha y su reentrenamiento. En Bamajian JV. ed. Terapeutica por el ejercicio. $3^{\mathrm{a}}$ ed. Buenos Aires: Panamericana, 1982: 211-225.

19.- Ericson MO, Nisell R, Ekholm J. Quantified electromyography of lower limb muscles. Scand J Rehab Med 1986; 18: 159-163.

20.- Lara MF, Angulo MT, Llanos LF. Actividad 
electromiográfica normal en la marcha humana. Biomecánica 1996 (IV); 7: 110-116.

21.- Pièra JB, Grossiord A. La marche. Encycl Med Chir. Paris, Kinésithérapie, 4.4.02, 26013; A-10 et A-15.

22.- Luttgens K, Wells K. Kinesiologia. Bases científicas del movimiento humano. Madrid: Pila Teleña, 1982.

23.- Joseph J, Watson R. Telemetering electromyography of muscles used in walfing up and down stairs. J Bone Joint Surg 1967; 49 (B): 774790 .

24.- Basmajian JV. Electrofisiología de la acción muscular. Buenos Aires: Panamericana, 1976.

25.- Battye CK, Joseph J. Investigation by telemetering of activity of some muscles in walking. Med Biol Eng 1966; 4: 125-134.

26.- Perry J. Gait Analysis. Normal and pathological funtion. Thorofare: Salck, 1992.

27.- Plas F, Viel E, Blanc Y. La marcha humana. Cinesiología dinámica, Biomecánica y patomecánica. Barcelona: Masson, 1984.

28.- Viladot Perice A, Viladot Voegeli A. La marcha humana. Rev Ortop Traum 1990;34 IB,1: 99-108.

29.- James B, Parker AW. Electromyography of stairs lococmotion in elderly men and women. Electromyogr Clin Neurophysiol 1989; 29: 161-168.

30.- Sanchez-Lacuesta J. Biomecánica de la marcha humana normal. En: Sanchez-Lacuesta J et al. eds. Biomecánica de la marcha humana normal y patológica. Valencia: Instituto de Biomecánica,1993: 19-112.
31.- Wooten ME, Kadaba MP, Cochran GVB. Dynamic electromyography I. Numerical Representation using principal component analysis. J Ortho Re1990; 8: 243-258.

32.- Wooten ME, Kadaba MP, Cochran GVB. Dynamic electromyography II. Normal patterns during gait. J Ortho Re1990; 8: 185-196.

33.- Andriacchi TP, Andersson GBJ, Fermier RW, Stern D, Galante JO. A study of lower-limb mechanics during stair-climbing. J Bone Joint Surg 1980; 62 (A): 749-756.

34.- Viladot A. Estudio de la marcha normal y patológica. En: Viladot R, Cohi O, Clavell S eds. Ortesis y Prótesis del Aparato Locomotor: Extremidad Inferior 2.1 Barcelona: Masson, 1987; 39-52.

35.- Porta J., Galiano D., Tejedo A., González Suso J.M. Valoración de la composición corporal. Utopías y realidades. En: Esparza Ros F. eds. Manual de cineantropometría. Monografías Femede: Grupo Español de Cineantropometría, 1993; 113-170.

36.- Sloan AW, Burt JJ, Blyth CS, Estimating body fat in young women. J Appl Physiol 1962; 17: 967.

37.- Ross WD, De Rose EH, Word R. Antropometría aplicada a la medición del deporte. En: Dirix A, Knuttpen NG, Tittel K. eds. Libro olímpico de la medicina del deporte. Doyma, 1988; 243-278.

38.- Izquierdo M., Angulo X. Estimación de la producción explosiva de fuerza: Consideraciones y tópicos. Arch Med Dep 1997; 62 (XIV): 493-503. 